\title{
Subclass Hierarchy on Circular Arc Bigraphs
}

\author{
Marina E. Groshaus, André L. P. Guedes, ${ }^{\circledR}$ Fabricio S. Kolberg
}

\begin{abstract}
Circular arc (CA) bigraphs, a bipartite variation of circular arc graphs, are bipartite graphs such that there exists a one-to-one correspondence between their vertices and a family of arcs on a circle such that vertices of opposite partite sets are neighbors precisely if their corresponding arcs intersect. In this paper, we explore the containment relationships between several subclasses of CA bigraphs, including circular convex bipartite (CCB) graphs, as well as the proper and Helly subclasses of CA bigraphs and interval bigraphs.
\end{abstract}

\section{Introduction}

Circular arc ( $C A)$ bigraphs are a bipartite variant of circular arc graphs. While their non-bipartite counterpart is a widely studied class of intersection graphs, circular arc bigraphs remain a relatively unexplored subject. Most results on the class and its subclasses are quite recent, and include matrix-based characterizations for the class and its proper and unit subclasses [1, efficient recognition algorithms for the proper [9] and Helly

2000 AMS Subject Classification: 05C62.

Keywords and Phrases: Graph classes; Circular arc bigraphs; Circular convex bipartite graphs

This research was supported by CAPES, CNPq and CONICET 
subclasses [3], and forbidden graph characterizations for some subclasses of the Helly subclass [4].

Interval bigraphs are a bipartite variation of interval graphs as well as a subclass of CA bigraphs. Being an object of study for at least three decades, characterizations and recognition algorithms for the class [8] and its proper [2] subclass have been presented, as well as studies on the relationship between proper interval bigraphs and several other classes of bipartite graphs [2] and between interval bigraphs and the complements of two-clique CA graphs [5].

In this paper, we present a full exploration of the containment relations between the class of CA bigraphs and several subclasses, including proper and Helly CA bigraphs, proper and Helly interval bigraphs, and circular convex bipartite $(C C B)$ graphs.

\section{Definitions}

We denote a bipartite graph as a triple $G=(X, Y, E)$ where $X, Y$ are its partite sets and $E$ is its edge set. For any $v \in V(G)$, we denote by $N(v)$ and $N[v]$ the open and closed neighborhoods of $v$, respectively.

We call vertices with the same open neighborhood twins, and say that a graph is twin-free if it contains no twins.

A bi-circular-arc model is a triple $(C, \mathbb{I}, \mathbb{E})$ such that $C$ is a circle, and $\mathbb{I}$ and $\mathbb{E}$ are families of arcs over $C$. The corresponding graph of a bi-circulararc model is constructed by creating one vertex corresponding to each arc of $\mathbb{I} \cup \mathbb{E}$, and edges between two vertices corresponding to $\operatorname{arcs} I \in \mathbb{I}, E \in \mathbb{E}$ precisely if $I \cap E \neq \emptyset$. A bipartite graph is a circular arc (CA) bigraph if and only if it is the corresponding graph of a bi-circular-arc model.

Similarly, a bi-interval model is a pair $(\mathbb{A}, \mathbb{B})$ of intervals on the Real line. The corresponding graph of a bi-interval model is constructed by creating a vertex for every element of $\mathbb{A} \cup \mathbb{B}$, and edges between vertices corresponding to two intervals $A \in \mathbb{A}, B \in \mathbb{B}$ precisely if $A \cap B \neq \emptyset$. A bipartite graph is an interval bigraph if it is the corresponding graph of a 
bi-interval model.

If a graph $G$ is the corresponding graph of some bi-circular-arc (biinterval) model, we say that $G$ admits the model. Note that, if a bipartite graph admits a model $(C, \mathbb{I}, \mathbb{E})$, then $\mathbb{I}$ corresponds to one partite set and $\mathbb{E}$ to the other. The same is true in bi-interval models.

If a graph $G$ admits some bi-circular-arc model $(C, \mathbb{I}, \mathbb{E})$, we denote by $a(v)$, for any $v \in V(G)$, the arc corresponding to $v$ in the model. Given some arc $A$ on a circle, denote by $s(A)$ and $t(A)$ its counter-clockwise and clockwise endpoints, respectively. Also, given two points $s, t$ on a circle, denote by $(s, t)$ an arc such that $s((s, t))=s$ and $t((s, t))=t$.

If $\left\{p_{1}, \ldots, p_{n}\right\}$ is a set of points on a circle $C$, we say that $\left(p_{1}, \ldots, p_{n}\right)$ is a clockwise order if a clockwise traversal of the circle starting at $p_{1}$ finds the points in that exact order.

A family of sets is said to be proper if no two elements of the family are contained in one another. A graph is a proper CA bigraph (proper interval bigraph) if it admits a bi-circular-arc model $(C, \mathbb{I}, \mathbb{E}$ ) (bi-interval model $(\mathbb{A}, \mathbb{B}))$ such that $\mathbb{I}$ and $\mathbb{E}(\mathbb{A}$ and $\mathbb{B})$ are proper families. It is easy to note that proper interval bigraphs are a proper subclass of proper $\mathrm{CA}$ bigraphs.

For a bipartite graph $G=(X, Y, E)$, a biclique is a maximal subset of $X \cup Y$ that induces a bipartite-complete graph. A Helly $C A$ bigraph is a graph that admits a bi-circular-arc model $(C, \mathbb{I}, \mathbb{E})$ such that, for every biclique $K$ in the graph, there is a point $p_{K} \in C$ such that every arc in $\mathbb{I} \cup \mathbb{E}$ that corresponds to a vertex of $K$ contains $p_{K}$. We call a model with that property a Helly bi-circular-arc model. Similarly, a Helly interval bigraph is a graph that admits a bi-interval model $(\mathbb{A}, \mathbb{B})$ such that, for any biclique $K$ in the graph, there is a number $n_{K}$ such that every interval in $\mathbb{A} \cup \mathbb{B}$ corresponding to a vertex of $K$ contains $n_{K}$. It is easy to note that Helly interval bigraphs are a proper subclass of Helly CA bigraphs.

A bipartite graph is bichordal if it admits no chordless cycle of length greater than four. A graph is a non-bichordal Helly CA bigraph if it is a Helly CA bigraph and is not bichordal. 
A bipartite graph $G=(X, Y, E)$ is a circular convex bipartite $(C C B)$ graph if $X$ (or $Y$ ) admits a circular order such that, for every vertex $w \in Y$ (or $v \in X$ ), the neighborhood of $w$ (or $v$ ) is an interval in the order. We call such an order a $C C B$ order. The class has been extensively studied for its computational properties [6, 7], but little is known about its structural properties and its relationship with other classes.

If a bipartite graph $G=(X, Y, E)$ is such that both $X$ and $Y$ admit their own CCB orders, then we say that it is doubly-CCB.

\section{Relationships between classes}

We start by situating the CCB and doubly-CCB classes with relation to CA bigraphs and the other subclasses we studied. Firstly, it is easy to verify that doubly-CCB is a proper subclass of $\mathrm{CCB}$, as Figure 1 contains an example of a CCB graph that is not doubly-CCB.

In the sequence, we show that CCB graphs are a proper subclass of CA bigraphs, and that all the other classes we study in this paper are proper subclasses of doubly-CCB.

Theorem 3.1. Every $C C B$ graph is a $C A$ bigraph.

Proof. Consider a graph $G=(X, Y, E)$ such that $X$ admits a CCB order $\left(v_{1}, \ldots, v_{n}\right)$. Construct a family of arcs corresponding to $X$ around some circle $C$ in the following way: make $n$ mutually disjoint arcs of the same size, and attribute them to the vertices of $X$ in the order given by the CCB order (i.e. $a\left(v_{1}\right)$ is immediately consecutive to $a\left(v_{2}\right)$, which is immediately consecutive to $a\left(v_{3}\right)$, and so on). Let $\mathbb{I}$ be the resulting family.

Given that $\left(v_{1}, \ldots, v_{n}\right)$ is a CCB order, note that it is possible to define, for all vertices $w \in Y$, an arc that intersects precisely the arcs corresponding to $N(w)$ in $\mathbb{I}$. Add those arcs to a family $\mathbb{E}$, and $(C, \mathbb{I}, \mathbb{E})$ is a bi-circular-arc model of $G$.

In Figure 1, we present a CA bigraph that is not CCB, showing that the containment in Theorem 3.1 is proper. 
We now show that every other class we cover is contained in doublyCCB. For that, it suffices to show that Helly CA bigraphs and proper CA bigraphs are both proper subclasses of doubly-CCB.

Theorem 3.2. If $G$ is a proper $C A$ bigraph, then it is doubly-CCB.

Proof. Let $G=(X, Y, E)$ be a proper CA bigraph with proper bi-circulararc model $(C, \mathbb{I}, \mathbb{E})$, with $\mathbb{I}$ representing set $X$ and $\mathbb{E}$ representing $Y$. Let $X=\left\{v_{1}, \ldots, v_{n}\right\}$ such that $\left(s\left(a\left(v_{1}\right)\right), s\left(a\left(v_{2}\right)\right), \ldots, s\left(a\left(v_{n}\right)\right)\right)$ is a clockwise order.

Since $\mathbb{I}$ is a proper family, we have that $\left(t\left(a\left(v_{1}\right)\right), t\left(a\left(v_{2}\right)\right), \ldots, t\left(a\left(v_{n}\right)\right)\right)$ is also a clockwise order. Consider, then, the circular ordering $\left(v_{1}, \ldots, v_{n}\right)$ of $X$. We show that every neighborhood in $Y$ is an interval of that ordering.

Suppose $w \in Y$ is such that $N(w)$ is not an interval in $\left(v_{1}, \ldots, v_{n}\right)$. Let $v_{a}, v_{b} \in N(w)$ with $a<b$ such that there is a vertex $v_{c} \notin N(w)$ with $c<a$ or $c>b$ and a vertex $v_{d} \notin N(w), a<d<b$. Note that $\left(s\left(a\left(v_{a}\right)\right), s\left(a\left(v_{d}\right)\right), s\left(a\left(v_{b}\right)\right), s\left(a\left(v_{c}\right)\right)\right)$ is a clockwise order, and that $a\left(v_{c}\right), a\left(v_{d}\right)$ must be disjoint, as otherwise, $a\left(v_{c}\right) \cup a\left(v_{d}\right)$ would contain either $a\left(v_{a}\right)$ or $a\left(v_{b}\right)$, making it impossible for $w$ to be neighbor to neither.

Consider the arcs $\left(t\left(a\left(v_{c}\right)\right), s\left(a\left(v_{d}\right)\right)\right)$ and $\left(t\left(a\left(v_{d}\right)\right), s\left(a\left(v_{c}\right)\right)\right)$ of the circle. We have that $a(w)$ must be entirely contained within one of them, since it must not intersect $a\left(v_{d}\right)$ nor $a\left(v_{c}\right)$. Suppose w.l.o.g. that $a(w)$ is in the former.

Note that $s\left(a\left(v_{b}\right)\right)$ is contained within the arc $\left(s\left(a\left(v_{d}\right)\right), s\left(a\left(v_{c}\right)\right)\right)$. If $a\left(v_{b}\right)$ intersects $a(w)$, then $t\left(a\left(v_{b}\right)\right)$ is contained in $\left(t\left(a\left(v_{c}\right)\right), s\left(a\left(v_{d}\right)\right)\right)$, which in turn implies $a\left(v_{b}\right)$ contains $a\left(v_{c}\right)$, leading to a contradiction. Therefore, every vertex $w \in Y$ is such that $N(w)$ is an interval in $\left(v_{1}, \ldots, v_{n}\right)$, implying it is a CCB order of $X$.

The proof that $Y$ admits a CCB order is analogous. Therefore, $G$ is doubly-CCB.

Theorem 3.3. If $G$ is a Helly $C A$ bigraph, then it is doubly-CCB.

Proof. Let $G=(X, Y, E)$ be a Helly CA bigraph, and $(C, \mathbb{I}, \mathbb{E})$ be a Helly bi-circular-arc model of $G$, with $\mathbb{I}$ corresponding to $X$, and $\mathbb{E}$ correspond- 
ing to $Y$. We may assume that $G$ is twin-free, as twins can be placed consecutively in a CCB order.

Note that, for every vertex $v \in X$, the set $N[v] \cup\left\{v^{\prime} \in X \mid N(v) \subseteq\right.$ $\left.N\left(v^{\prime}\right)\right\}$ is a biclique in $G$. Call that biclique $K_{v}$ for all $v \in X$. By the definition of a Helly model, we have that in $(C, \mathbb{I}, \mathbb{E})$, there exists a point $p_{v}$ that all arcs corresponding to vertices in $K_{v}$ contain, for all $v \in X$. Let $X=\left\{v_{1}, \ldots, v_{n}\right\}$ such that $\left(p_{v_{1}}, p_{v_{2}}, \ldots, p_{v_{n}}\right)$ is a clockwise order. We show that $\left(v_{1}, \ldots, v_{n}\right)$ is a CCB order.

Suppose $w \in Y$ is such that $N(w)$ is not an interval in $\left(v_{1}, \ldots, v_{n}\right)$. Note that, for all $v \in N(w), w \in K_{v}$, and therefore $p_{v} \in a(w)$. Let $v_{a}, v_{b}, v_{c}, v_{d} \in X$ such that $v_{a}, v_{c} \in N(w), v_{b}, v_{d} \notin N(w)$ and $a<b<c<d$ or $d<a<b<c$ (suppose the former w.l.o.g.). Note that the $\operatorname{arc}\left(p_{v_{a}}, p_{v_{c}}\right)$ on the circle contains $p_{v_{b}}$, and the arc $\left(p_{v_{c}}, p_{v_{a}}\right)$ on the circle contains $p_{v_{d}}$. Also note that $a(w)$ must contain one of these two arcs, as otherwise it cannot intersect both $p_{v_{c}}$ and $p_{v_{a}}$. In that case, however, $a(w)$ must contain either $p_{v_{b}}$ or $p_{v_{d}}$, leading to a contradiction.

The proof that $Y$ also admits a CCB order is analogous. Therefore, $G$ is doubly-CCB.

Therefore, Helly CA bigraphs and proper CA bigraphs are both doublyCCB. Once again, Figure 1 contains example graphs that show the containments are proper. Furthermore, the proof that Helly CA bigraphs and proper CA bigraphs are not comparable is also in Figure 1, as it contains examples of a proper CA bigraph that is not Helly, and a Helly CA bigraph that is not proper.

The relationships that remain to be explored are shown in detail in [4], where the following couple of theorems are proven.

Theorem 3.4. [4] Every Helly interval bigraph is a proper interval bigraph.

Theorem 3.5. [4] Every non-bichordal Helly CA bigraph is a proper CA bigraph. 
The fact that Helly interval bigraphs are proper interval bigraphs, and that non-bichordal Helly CA bigraphs are proper CA bigraphs, provides us with some insight on the kinds of graphs that lie in the intersection between proper and Helly CA bigraphs. It does not, however, lead to a full characterization of that intersection, which remains an open problem.

And with that, the exploration of the containment relations between the classes presented is complete. In the sequence, we present a Venn diagram of the studied classes, with an example graph for every region.

\section{Containment Diagram}

In Figure 1, we present a Venn diagram of the studied classes. In the diagram, $\mathbf{P}$ and $\mathbf{H}$ stand for proper and Helly CA bigraphs, respectively, PI and HI for proper and Helly interval bigraphs respectively, $\mathbf{N B H}$ for non-bichordal Helly CA bigraphs, CCB and D-CCB for CCB and doubly-CCB graphs respectively, and $\mathbf{C A B}$ for $\mathrm{CA}$ bigraphs. Note that the figure also presents example graphs for every region.

\section{Conclusion}

We provided a comprehensive study of the relationship between seven different subclasses of circular arc bigraphs. The results provide a full understanding of the containment hierarchies of the classes mentioned, allowing us to present a comprehensive diagram of them. The classes presented in this paper, however, are not an exhaustive list of relevant subclasses of CA bigraphs, and several other subclasses are yet to have their containment relations explored.

Future research includes covering other classes, such as unit CA bigraphs, normal CA bigraphs, and others. It also includes attempting to characterize the exact class of graphs that lies in the intersection between proper and Helly CA bigraphs. 


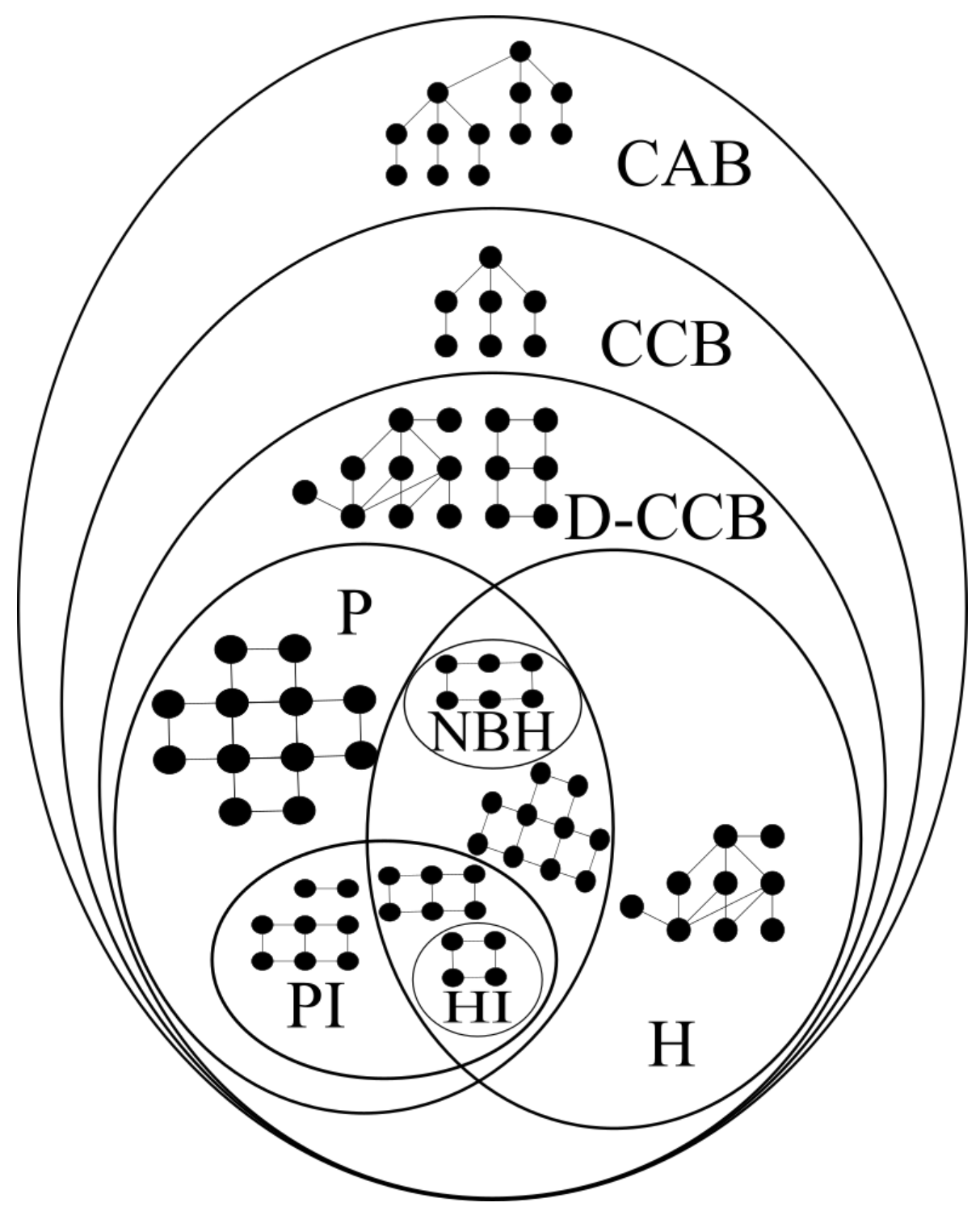

Figure 1: A Venn diagram of the presented classes. 


\section{References}

[1] A. Basu, S. Das, S. Ghosh and M. Sen, Circular-arc bigraphs and its subclasses. Journal of Graph Theory 73 (2013), no. 4, 361 - 376.

[2] D. E. Brown and J. R. Lundgren, Characterizations for unit interval bigraphs. Congressus Numerantium 206 (2010), 5 - 17.

[3] M. E. Groshaus, A. L. P. Guedes and F. S. Kolberg, Subclasses of circular-arc bigraphs: Helly, normal and proper. Electronic Notes in Theoretical Computer Science 346 (2019), 497 - 509.

[4] M. E. Groshaus, A. L. P. Guedes and F. S. Kolberg, On the Helly subclasses of interval bigraphs and circular arc bigraphs. In Proceedings of the 14th Latin American Theoretican Informatics Symposium (2020), São Paulo - SP, Brazil.

[5] P. Hell and J. Huang, Interval bigraphs and circular arc graphs. Journal of Graph Theory 46 (2004), 313 - 327.

[6] Y.D. Liang and N. Blum. Circular convex bipartite graphs: Maximum matching and Hamiltonian circuits. Information Processing Letters 56 (1995), no. 4, 215 - 219.

[7] T. Liu, M. Lu, Z. Lu and K. Xu. Circular convex bipartite graphs: Feedback vertex sets. Theoretical Computer Science 556 (2014), 55 62.

[8] A Rafiey, Recognizing interval bigraphs by forbidden patterns. ArXiv (2012), abs/1211.2662.

[9] M. Safe, Circularly compatible ones, D-circularity, and proper circular-arc bigraphs. ArXiv (2019), abs/1906.00321. 


$\begin{array}{ll}\text { Marina Esther Groshaus } & \text { André Luiz Pires Guedes } \\ \text { Curitiba - PR, Brazil } & \text { Curitiba - PR, Brazil } \\ \begin{array}{l}\text { Departamento Acadêmico de In- } \\ \text { formática - Universidade Tec- }\end{array} & \begin{array}{l}\text { Departamento de Informática - } \\ \text { nológica Federal do Paraná }\end{array} \\ \text { marinagroshaus@utfpr.edu.br } & \text { andre@inf.ufpr.br }\end{array}$

Fabricio Schiavon Kolberg

Curitiba - PR, Brazil

Departamento de Informática -

Universidade Federal do Paraná

fskolberg@inf.ufpr.br 